\title{
Frequency Domain Optical Coherence Tomography Techniques in Eye Imaging
}

\author{
M. Wojtkowski, A. KowalczyK*, P. Targowski \\ AND I. GORCZYŃSKA \\ Institute of Physics, Nicholas Copernicus University \\ Grudziądzka 5, 87-100 Toruń, Poland
}

(Received June 3, 2002; in final form September 27, 2002)

\begin{abstract}
This contribution presents an application of frequency-domain optical tomography to ophthalmology. Essential theoretical foundations of time-domain and frequency-domain optical tomography are presented. Images of sections through the anterior chamber, the corneo-scleral angle and fundus of the eye are reconstructed from the spectral fringes. The morphological information gained by tomograms is important for diagnosing and planning of a treatment of glaucoma.
\end{abstract}

PACS numbers: 42.30.Wb, 42.66.Ew, 42.66.-p, 07.60.-j, 07.60.Ly

\section{Introduction}

In optical coherence tomography (OCT), one detects light scattered back from an object that is illuminated by a light source of low temporal but high spatial, coherence. The narrow beam of such light penetrates the object, scatters from the elements of its structure and is brought to interference with a reference beam by the use of an interferometer. Because of low temporal coherence of the light the interference can occur only when the optical paths of the reference and scattered beams are equal to within the range of the longitudinal coherence length. Therefore, if an optical delay line is employed to change the optical path of the reference beam, the depth position of the scattering centre is easily obtained from the corresponding position of a reference mirror on registration of the interference signal.

*corresponding author; e-mail: akowal $@_{\text {phys.uni.torun.pl }}$ 
Some improvements in OCT technique have been made to eliminate moving parts, shorten the collection time, enhance signal-to-noise ratio and make the system insensitive to low frequency oscillations. One of the most promising new implementation is frequency-domain OCT [1]. Here, information on the distribution of the scattering centres along the path of the probing beam is obtained simply by the Fourier transform of the interference pattern superimposed on a spectrum of the light produced at the output of the interferometer. Frequency-domain OCT requires a spectrometer (a grating) to produce a spectrum, and a CCD camera to register it. As it does not require moving parts it potentially could be faster than time-domain OCT.

At present, the temporal OCT technique dominates. The frequency-domain technique is less mature and therefore, so far, less popular in application. However, the elimination of moving parts integral to the measurement [2], the short collection time, and the inherently direct access to spectral information [3], all render this technique advantageous in comparison with temporal OCT. The depth range, which basically depends on spectral resolution, may be extended by application of a so-called complex analysis [4]. Fourier spectral radar is a technique originating from frequency-domain OCT. It measures the profile of a skin surface, as first demonstrated by Häusler and Lindner [5].

The most advanced application of OCT is in ophthalmology, where imaging studies have demonstrated significant potential for routine clinical examination of the anterior chamber of the eye, of the crystalline lens, and of the retina [6].

In the present contribution, the technological implementation of frequency-domain OCT to ophthalmology is presented.

\section{Theory}

The basic technique of the OCT is partial coherence interferometry, i.e. interference of broadband light waves. A beam originating from a light source is split into two partially coherent beams. One beam penetrates an object along the $z$-axis, and is scattered from the $i$-th scattering centre. The optics ensure that only the fraction which is scattered back along the $z$-axis is transmitted back to the interferometer. The complex representation of the polichromatic light wave $u_{i}$ scattered from the object is following:

$$
u_{i}=u\left(t+\tau_{i}\right)=\int_{-\infty}^{+\infty} U_{i}(\nu) \exp \left[-2 \pi \mathrm{i} \nu\left(t+\tau_{i}\right)\right] \mathrm{d} \nu
$$

where $U_{i}(\nu)$ is a complex amplitude.

The complex representation of the beam reflected from the reference mirror is

$$
u_{\mathrm{r}}=u\left(t+\tau_{\mathrm{r}}\right)=\int_{-\infty}^{+\infty} U_{\mathrm{r}}(\nu) \exp \left[-2 \pi \mathrm{i} \nu\left(t+\tau_{\mathrm{r}}\right)\right] \mathrm{d} \nu
$$


These two beams are delayed by $\tau_{i}=2 z_{i} / c$ and $\tau_{\mathrm{r}}=2 z_{\mathrm{r}} / c$, respectively. Resulting interference is registered by a photodetector.

In order to make theoretical equations more comprehensive we assume one state of polarisation and equal intensities of light reflected from the reference mirror and scattered back from an object.

\subsection{Time-domain $O C T$}

The information on the position of scattering centres in temporal OCT derives directly from the equation describing the interference of two beams

$$
I\left(\tau_{i}-\tau_{\mathrm{r}}\right)=I_{\mathrm{r}}+I_{i}+\operatorname{Re}\left\{\Gamma\left(\tau_{i}-\tau_{\mathrm{r}}\right)\right\}
$$

where $I_{\mathrm{r}}=\left\langle u\left(t+\tau_{\mathrm{r}}\right) u^{*}\left(t+\tau_{\mathrm{r}}\right)\right\rangle$ and $I_{i}=\left\langle u\left(t+\tau_{i}\right) u^{*}\left(t+\tau_{i}\right)\right\rangle$ are the intensities of light scattered at the $i$-th centre and reflected from the mirror, respectively, and $\Gamma\left(\tau_{i}-\tau_{\mathrm{r}}\right) \equiv\left\langle u\left(t+\tau_{\mathrm{r}}\right) u^{*}\left(t+\tau_{i}\right)\right\rangle$ is a first-order electric field correlation function.

It is clear from Eq. (3) that interference will be registered if the delay $\tau_{\mathrm{r}}$ introduced by a mirror displacement is equal to $\tau_{i}=2 z_{i} / c$ within the coherence length.

\section{D. Frequency-domain OCT}

In frequency-domain OCT, the interferogram is measured as a function of frequency $[7]$ :

$$
\begin{aligned}
& G_{U U}(\nu)=G_{U_{\mathrm{r}} U_{\mathrm{r}}}(\nu)+\sum_{i} G_{U_{i} U_{i}}(\nu)+2 \operatorname{Re}\left[\sum_{i \neq j} G_{U_{i} U_{j}}(\nu)\right] \\
& +2 \operatorname{Re}\left[\sum_{i} G_{U_{\mathrm{r}} U_{i}}(\nu)\right],
\end{aligned}
$$

where $G_{U_{m} U_{n}}(\nu) \equiv \lim _{T \rightarrow \infty}\left[\frac{\overline{U_{m}(\nu) U_{n}^{*}(\nu)}}{2 T}\right]$ is called cross-spectral density function [8], $U_{\mathrm{r}}(\nu) \equiv \int_{-\infty}^{+\infty} u\left(t+\tau_{\mathrm{r}}\right) \exp (2 \pi \mathrm{i} \nu t) \mathrm{d} t=\mathrm{FT}^{-1}\left[u\left(t+\tau_{\mathrm{r}}\right)\right]$ is the Fourier transform of the light wave reflected from the reference mirror, and $\sum_{i} U_{i}(\nu) \equiv$ $\sum_{i} \int_{-\infty}^{+\infty} u\left(t+\tau_{i}\right) \exp (2 \pi \mathrm{i} \nu t) \mathrm{d} t=\sum_{i} \mathrm{FT}^{-1}\left[u\left(t+\tau_{i}\right)\right]$ originates from light waves backscattered along the penetrating beam within the object. Therefore, a Fourier transform of the cross-spectral density function $G_{U U}(k)$ yields

$$
\begin{gathered}
\mathrm{FT}^{-1}\left[G_{U U}(\nu)\right]=\mathrm{FT}^{-1}\left[G_{U_{\mathrm{r}} U_{\mathrm{r}}}(\nu)\right]+\sum_{i} \mathrm{FT}^{-1}\left[G_{U_{i} U_{i}}(\nu)\right] \\
+2 \sum_{i \neq j} \mathrm{FT}^{-1}\left[G_{U_{i} U_{j}}^{\mathrm{R}}(\nu)\right]+2 \sum_{i} \mathrm{FT}^{-1}\left[G_{U_{\mathrm{r}} U_{i}}^{\mathrm{R}}(\nu)\right],
\end{gathered}
$$

where superscript $\mathrm{R}$ denotes the real part of $G$. 
According to the Wiener-Khinchin theorem [7], there is a correspondence between a truncated real spectrum and the complex first-order electric field correlation function, $\Gamma$ :

$$
\Gamma(\tau)=4 \int_{0}^{+\infty} G_{U_{1} U_{2}}^{\mathrm{R}}(\nu) \exp (-2 \pi \mathrm{i} \nu t) \mathrm{d} \nu .
$$

Applying this theorem to Eq. (5), one obtains

$$
\begin{aligned}
& \mathrm{FT}^{-1}\left[G_{U U}(\nu)\right]=\Gamma_{\mathrm{r}}(\tau)+\sum \Gamma_{i}(\tau)+\sum_{i \neq j} \Gamma\left(\tau+\tau_{j}-\tau_{i}\right)+\sum_{i \neq j} \Gamma\left(\tau+\tau_{i}-\tau_{j}\right) \\
& \quad+\sum_{i} \Gamma\left(\tau+\tau_{i}-\tau_{\mathrm{r}}\right)+\sum_{i} \Gamma\left(\tau+\tau_{\mathrm{r}}-\tau_{i}\right) .
\end{aligned}
$$

The function described by Eq. (7) is symmetrical about $\tau=0$ because it represents a Fourier transform of a real function. Two first terms produce intense peaks at $\tau=0$. The two next depend on mutual positions of scattering centres within the object and do not convey any useful information on the structure of the object. Both groups may be regarded as parasitic ones. Two last terms in Eq. (7) provide direct information on the positions of scattering centres with respect to the (constant) position of the reference mirror. It may be noted that the position of the first four terms on the $\tau$ scale does not depend on the position of the reference mirror while the last two terms by definition depend on the position of the reference mirror.

All terms mix, which deteriorates signal-to-noise ratio and obscures the interpretation of the results. Therefore the most important task is to extract two last terms which contain direct information on the positions of scattering centres. The different dependence of parasitic and useful terms on the reference mirror position allows to extract the useful signal. In the simplest method the reference mirror is shifted by a distance larger than $\max \left(z_{i}-z_{j}\right)$ causing that the terms containing useful information are separated from the obscuring ones. Such a procedure reduces a useful measured range which is especially severe in the case of the objects of considerable thickness. Another method - so-called complex method [4] — allows to calculate the phase $\phi(\nu)$ and amplitude $a(\nu)$ of complex $G_{U_{i} U_{\mathbf{r}}}(\nu)$ from the five consecutive measurements of $G_{U U}(\nu)$ at five different positions of the reference mirror. Finally, the $\mathrm{FT}^{-1}\{a(\nu) \exp [i \phi(\nu)]\}$ yields pure structural information of the object.

\section{Method}

The OCT instrument is based on the Michelson interferometer setup (Fig. 1). The light source is a superluminescent diode emitting at $810 \mathrm{~nm}$ with a $20 \mathrm{~nm}$ FWHM (Superlum, Moscow); for detailed description of the superluminescent diodes see [9]. The reference mirror is placed on the piezo-translator (Fig. 1a) for the complex method. The detection unit consists of a diffraction grating 
(1800 grooves $/ \mathrm{mm}$ blazed at $\lambda_{\mathrm{b}}=800 \mathrm{~nm}$ ) and a 16-bit CCD camera (Andor Technologies). A helium-neon laser serves for beam adjustment. The software, which controls the equipment and analyses the data, is written in the Lab-View environment.
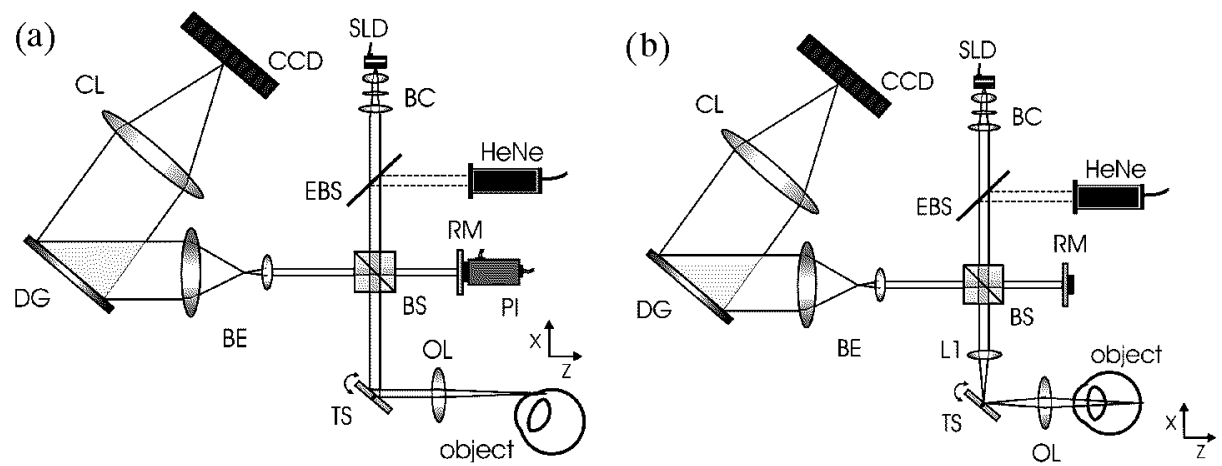

Fig. 1. Optical scheme of (a) complex frequency-domain OCT device used in corneo-scleral angle measurement, (b) frequency-domain OCT applied to measure a fundus: SLD - superluminescent diode, BC - collimator, EBS - edge beam splitter, HeNe - helium-neon laser, RM - reference mirror, PI - piezo-element, BS - beam splitter, OL - objective lens, L1 - lens, TS - transversal scanner, BE - beam expander, DG - diffraction grating, CL - camera objective, CCD - camera.

In order to create images of the corneo-scleral angle it was necessary to rotate the optical axis of the eye approximately $30^{\circ}$ from the optical axis of the instrument. The transverse scanner TS and the cornea were placed at the focal points of the object lens OL $(f=80 \mathrm{~mm})$. Such design ensures that the direction of the beam is insensitive to the scanner angle. The optical system produces relatively small probing spot size $(\Delta x \approx 35 \mu \mathrm{m})$ together with relatively long depth of focus $\Delta z \approx 3 \mathrm{~mm}$.

For imaging of the retina the imaging system employs the lens L1 $(f=$ $80 \mathrm{~mm})$, which focuses the beam on the scanner and the object lens OL $(f=$ $12 \mathrm{~mm}$ ). These two lenses form a telescope producing a parallel beam, which is focused on the retina by an optical system of the eye. The arrangement of the optical system guarantees that the scanning beam enters the eye at different angles but hits the same point at the pupil. The spot size and depth of focus are $\Delta x \approx 10 \mu \mathrm{m}, \Delta z \approx 0.5 \mathrm{~mm}$, respectively. In order to increase the signal-to-noise ratio all lenses were coated with antireflection layers. In both cases the incident power was approximately $250 \mu \mathrm{W}$.

The dynamic range $67 \mathrm{~dB}$ was estimated experimentally as the ratio of the optical power corresponding to camera saturation to the optical power, at which interference fringes were still distinguishable from the noise. 


\section{Results and discussion}

The tomogram of the anterior chamber of a porcine eye is presented in Fig. 2. The image consists of 700 vertical lines. Here the complex method was used to extract the useful information from the total signal. As a result, a depth of $0.4 \mathrm{~cm}$ in the axial direction, twice more than for the conventional frequency-domain technique, was obtained. Such clear reproduction of a corneal profile may be helpful in planning and following up keratotomy operations. The anatomy of the corneo-scleral angle is partially obscured by the nontransparent sclera.
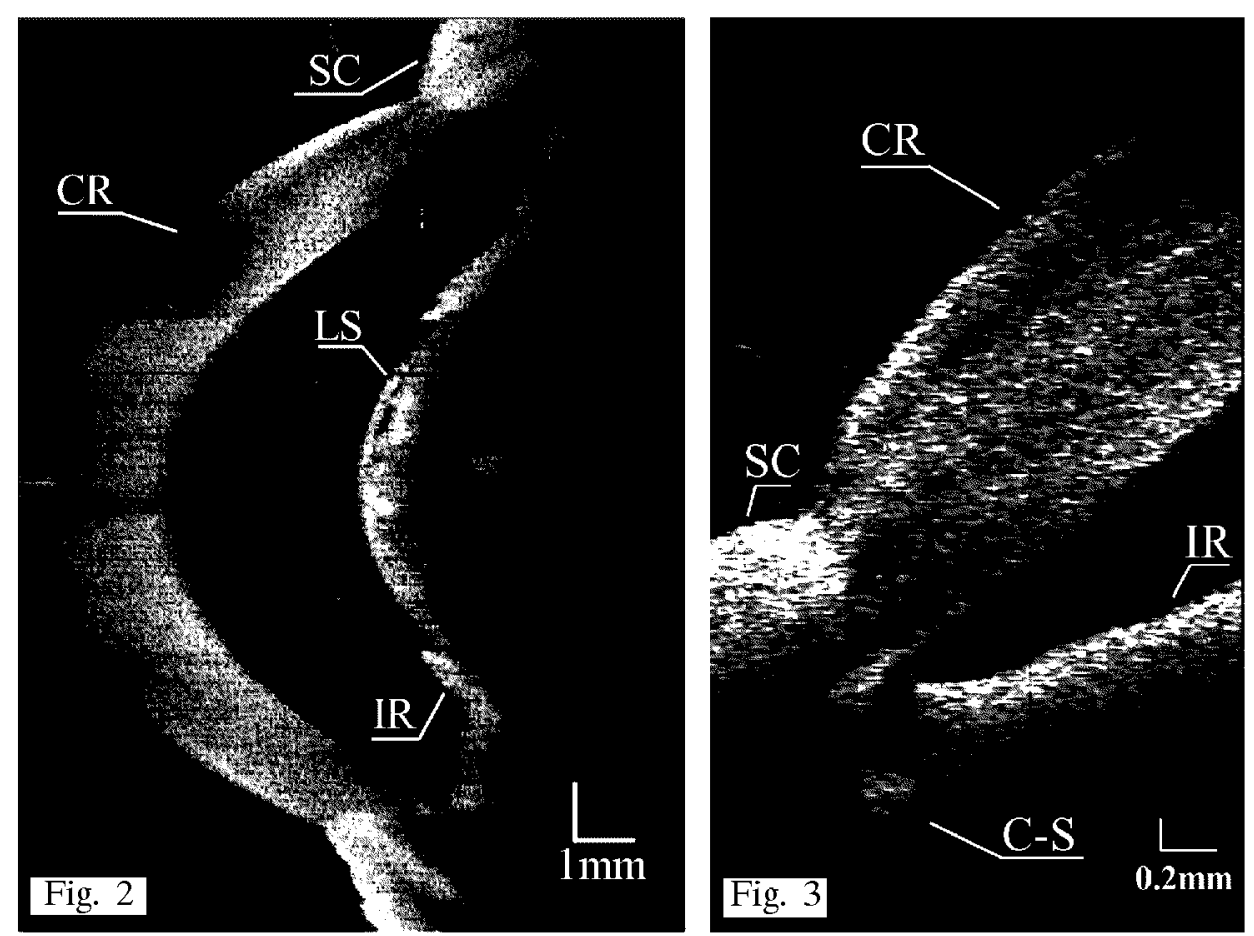

Fig. 2. Optical tomogram of the anterior chamber of a pig eye in vitro. CR - cornea, $\mathrm{SC}$ - sclera, LS - lens, IR - iris.

Fig. 3. Optical tomographic image of the corneo-scleral angle of a pig eye in vitro. CR - cornea, SC - sclera, IR - iris, C-S - corneo-scleral angle region.

In order to get an image of details of the corneo-scleral angle hidden under sclera, the penetrating beam was directed at an angle of approximately $30^{\circ}$ to the optical axis of the eye. The image (Fig. 3) exhibits more details but due to refraction the geometry of the image considerably differs from that presented at Fig. 2. Information provided here might be instrumental in planning and performing trabeculoplasty. 


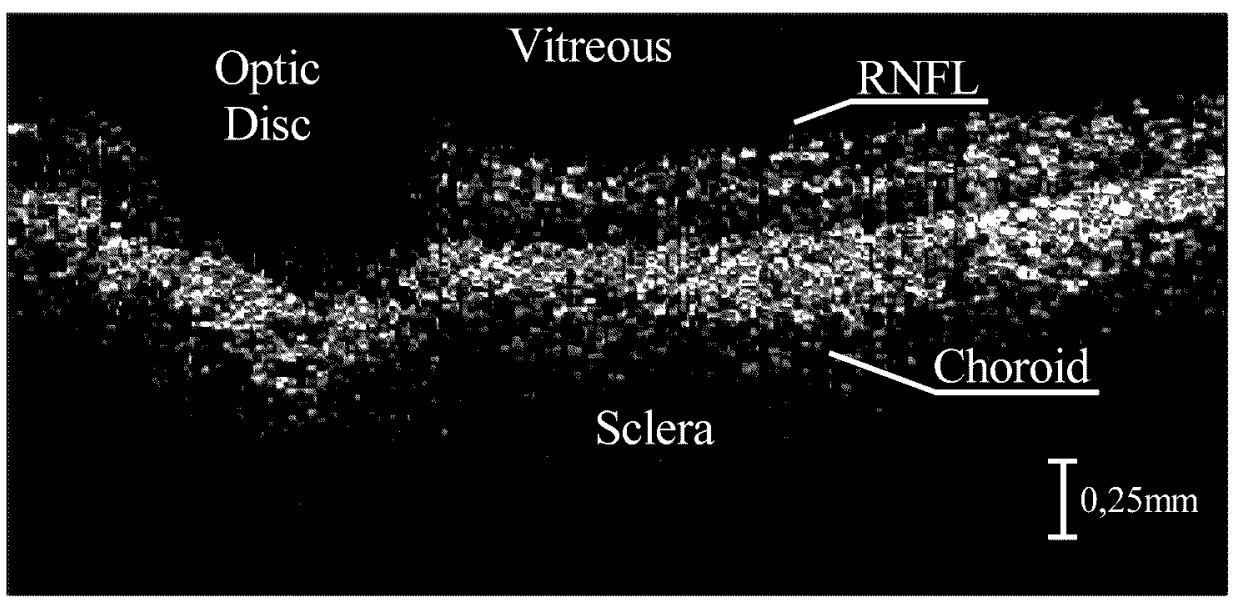

Fig. 4. Frequency-domain OCT tomogram of part of a pig eye fundus in vitro, RFNL - retinal nerve fibre layer.

Figure 4 shows the tomogram of the retina of the porcine eye. The information on the position of the scattering centres was separated from the total signal by an appropriate shift of the reference mirror. The border between the vitreous and the surface of the retina is sharp and the topography of optic disc is visible. As structural parts of the retina do not differ in refractive index, its internal structure, as revealed by OCT, is considerably blurred. Nevertheless, it is possible to estimate the thickness of the nerve fibre layer - the essential parameter in diagnosis of glaucoma. The visibility of anatomical details depends on the rate of change of refractive index along the interrogating beam. If it changes abruptly, as at the cornea-air, cornea-liquid, vitreous-retina interface, the image is sharp and clear. If the refractive index varies gently, as in case of the retina, the image is blurred. Obviously, if there is strong absorption or scattering, the photons are lost and the image cannot be reconstructed to the desired depth. For weakly absorbing and weakly scattering media of the anterior chamber the penetration is limited only by spectral resolution, while for the retina is merely $0.6 \mathrm{~mm}$.

It should be noted that the dimensions in OCT tomograms are in fact products of refractive index and geometrical dimensions. In order to obtain real geometry, therefore, the image must be corrected by taking refraction into account. The need of such correction is evident when two tomograms collected at different angles are compared.

In our original setup the rate determining process was data transmission between CCD and PC which caused that it took about 1.5 minutes to collect the data necessary to reconstruct the image composed of 700 lines (like in Fig. 2), the recent improvements in the CCD driver has shortened this time to 2 seconds not unacceptably long for a measurement in human patients. Our goal is to achieve 
at least $100 \mu$ s per A-scan in order to perform real time retinal OCT imaging. This will influence patient's comfort and it will make the method less sensitive for accidental eye and head movement, which results in improvement of the quality of retinal tomograms.

\section{Conclusions}

This work presented in this paper demonstrates the potential diagnostic value of the OCT and indicates the advantages to be gained from the frequencydomain method as compared with the time-domain version.

\section{Acknowledgment}

This work was supported by the grant no. 4T11E 02322 of the State Committee for Scientific Research.

\section{References}

[1] A.F. Fercher, C.K. Hitzenberger, G. Kamp, S.Y. El-Zaiat, Opt. Commun. 117, $43(1995)$.

[2] A.F. Zuluaga, R. Richards-Kortum, Opt. Lett. 24, 519 (1999).

[3] R. Leitgeb, M. Wojtkowski, C.K. Hitzenberger, M. Sticker, A. Kowalczyk, A.F. Fercher, Opt. Lett. 25, 820 (2000).

[4] A.F. Fercher, R. Leitgeb, C.K. Hitzenberger, H. Sattmann, M. Wojtkowski, Proc. SPIE 3564, 173 (1999).

[5] G. Häusler, M.W. Lindner, J. Biomed. Opt. 3, 21 (1998).

[6] M.R. Hee, J.A. Izatt, E.A. Swanson, D. Huang, J.S. Schuman, C.P. Lin, C.A. Puliafito, J.G. Fujimoto, Arch. Ophthalmol. 113, 325 (1995).

[7] J.W. Goodman, Statistical Optics, Wiley, New York 1985.

[8] M. Born, E. Wolf, Principles of Optics, 7 th ed., Cambridge Univ. Press, Cambridge 1999 , p. 567.

[9] A.T. Semenov, V.K. Batovrin, I.A. Garmash, V.R. Shidlovski, M.V. Shramenko, S.D. Yakubowich, Electr. Lett. 31, 314 (1995). 